\title{
Subject Index Vol. 2, 2005
}

\section{Neurn denenerative Diseases}

Acetylcholinesterase 16

Activity-dependent neurotrophic factor 135

Adenosine $\mathrm{A}_{2 \mathrm{a}}$ receptor 160

ALS, clinical trials 215

-, translational research 215

ALS2 gene 135

Alsin, anti-ALS activity 135

Alzheimer disease, cerebrospinal fluid 242

Alzheimer's disease 28, 36, 36, 220, 255, 267, 284, 299, 305

AMPA receptors 147

Amyloid 261, 273

- beta precursor protein 277

- - protein 36

- precursor protein 284

- $\beta 1-38242$

- $\quad-40242$

- - -42242

$\beta$-Amyloid 28, 233

Amyloidosis 284

Amyotrophic lateral sclerosis $115,128,135,139,160,177$, $195,202,246$

- - - etiology, environmental factors 195

- $\quad$ - , prognostic factors 208

- $\quad$ - , toxicity pathways 166

Apolipoprotein E 28

Astrocyte-motor neuron interactions 139

A $\beta$ immunization 267

- immunotherapy 255

B cells 267

BACE 284

Bovine spongiform encephalopathy 1

Brain 6

Brain-derived neurotrophic factor 160

$\mathrm{Ca}^{2+}$ metabolism 147

Celastrol 246

Cerebral amyloid angiopathy 305
Cholesterol 233

- 25-hydroxylase 233

Clinical trials in ALS 208

Cognitive impairment 56

Congophilic angiopathy 261

Copper-zinc superoxide dismutase 115,135

Creutzfeldt-Jakob disease 1

Dementia 56, 233, 273

Differential scanning calorimetry 115

Diffusion tensor imaging 177

DJ-1 313

Dynein/dynactin 215

Elevated plasma homocysteine levels 299

Enzyme-linked immunosorbent assay 242

Excitotoxicity 147

Fibroblast growth facotr 139

Frontotemporal dementia 28

Gene silencing 220

- therapy 220

Genetic association 233

Heat shock protein 70246

Hemorrhage 261

Hippocampus 6, 273

Huntington's disease 220

Hypermetabolism 202

Hyperostosis frontalis interna 56

Immunization 273

Immunohistochemistry 44

Immunotherapy 36, 267

Interneurons 16

Ionotropic glutamate receptor 49

Knock-in mouse model 6

Kunitz protease inhibitor 277

Kynurenine pathway 166

Lathosterol 233

Lead exposure 195
Lipoprotein receptor-related protein 277

Magnetic resonance imaging, gradient echo 305

Mammalian reticulons 185

- - functions 185

- - , structure 185

1-Methyl-4-phenyl-1,2,3,6tetrahydropyridine 44

Microbleed 305

Middle cerebral artery occlusion 49

Monkey 313

Monozygotic 56

Morgagni-Stewart-Morel 56

Motoneurons 16

Motor neuron degeneration 147

- - disease(s) 115, 215

- $\quad$ - (s), experimental

Mouse 313 therapies 215

Nerve growth factor 139, 277

Neurodegeneration 1

Neurofibrillary tangles 28

Neuroimaging 305

Neuroinflammation 128, 246

Neuropsychology 56

Nitric oxide production in motor neurons 128

Notch1 277

Obesity 56

p38 mitogen-activated protein kinase 128

Parkinson's disease 28, 44, 220

Passive immunization 36

Phase angle 202

Presenilin 6

Prions 1

Proinflammatory cytokines 128

Protein misfolding 115

Proteomics of the cerebrospinal fluid 177

Quinolinic acid 166
Reactive oxygen species 128

Reticulons as disease markers 185

Reverse genetics 220

RNA interference 220

Saitohin 28

$\gamma$-Secretase 6

Seizure 56

Senile plaques 28

Silent brain infarctions 299

SOD1 mutants 128

Spatial memory 6

Striatum 313

Substantia nigra 313

Susceptibility gene 233

$\alpha$-Synuclein 313

T cells 267

Tau 233

TAU haplotype 28

Tau protein 28

Thrombolysis 49

Transcranial magnetic stimulation 177

Transgenic mice 261

- mouse 284

Trk receptor transactivation 160

Tumor necrosis factor-alphaconverting enzyme 277

- - factor- $\alpha 128$

Twins 56

Vaccination 273

Water maze task 6 